\title{
Granjas modelos, granjas experimentales y enseñanza de la agricultura en Cádiz, 1855-1888: Historia de un fracaso
}

\begin{abstract}
ANTONIO CABRAL CHAMORRO
Independientemente de las matizaciones que estemos dispuestos a realizar, las páginas que escribiera Vicens Vives sobre la evolución secular de la agricultura española de los siglos XIX y primer tercio del XX siguen aún vivas.

Para Vicens; a partir de los datos de Salvador Millet, la reforma agraria liberal trajo un "considerable proceso de expansión agrícola" con la puesta en cultivo de varios millones de hectáreas entre 1818 y 1860. El siguiente período (1860-1900) fue de "reajuste": retroceso de los cereales con aumento de rendimientos y avance del viñedo, el olivar y los frutales. De toda esta historia conviene destacar dos cuestiones: en primer lugar, no escapó a nuestro autor la dirección del progreso agrario y sus dificultades y, en segundo lugar, si bien señaló como protagonistas del cambio agrario a los propietarios particulares, no dejó de apuntar el posible papel que en la segunda mitad del siglo XIX y primer tercio del XX pudiera haber jugado el Estado mediante la enseñanza agraria y el establecimiento de centros de experimentación y de asesoramiento agropecuario (1). Sin embargo, la historiografía española eligió uno de los posibles derroteros: obsesionada por el atraso secular de España respecto a Europa se embarcó en una larga aventura a la búsqueda de las causas del atraso.

Mediada la década de los ochenta el panorama comenzó a variar sustancialmente. Un grupo de investigadores, entre quienes destacaron los componentes de GEHR (2), puso de manifiesto que no todo en la agricultura española del último tercio del XIX y primer tercio de nuestro siglo XX fue inmovilismo y rutina $\mathrm{y}$, en la medida en que esta tesis se fue abriendo paso, quedó al descubierto un nuevo campo para la investigación, esto es, los factores del progreso agrario. Si hasta ayer una de las estrellas historiográficas fueron los factores del atraso, hoy comienzan a tener predicamento los factores del progreso.

Pues bien, entre los factores del progreso agrario de la agricultura española ha sido señalado de un modo especial el papel que el Estado comenzó a asumir

(1) J. VICENS VIVES (1972), PP. 584-587.

(2) D. GALLEGO (1986); J.I. JIMENEZ BLANCO (1986 a); S. ZAPATA (1986) y J.F. ZAMBRANA PINEDA (1987). Como síntesis: R. GARRABOU y J. SANZ (1985); J.I. JIMENEZ BLANCO (1986 b) y J. SANZ (1987).
\end{abstract}

DOI: http://dx.doi.org/10.25267/Trocadero.1995.i6.09 
a finales del siglo XIX en la difusión y divulgación de novedades agropecuarias y por supuesto en la creación de granjas-experimentales y estaciones de todo tipo (3). El "atraso" puede seguir existiendo, pero ahora es obligado incluir la mayor o menor presencia y/o ausencia del Estado como una de sus claves explicativas.

Por mi parte, coincido con todos aquellos que sostienen el relativo progreso de la agricultura española -y gaditana- y con aquellos otros que señalan el papel que en este progreso desempeñó el Estado.

Efectivamente, a finales de 1888 se instaló en Jerez la Granja-Escuela de Experimentación, iniciándose así la andadura de una de las instituciones más importantes para el desarrollo de la economía agraria gaditana y aun de la andaluza, hasta el punto de que sin la granja-experimental jerezana resultaría muy difícil de entender la reconstitución de los viñedos filoxerados, la mecanización, la introducción de los abonos químicos, la selección de semillas e introducción de nuevas variedades y un largo etcétera al que espero poder ocuparme y hacer justicia en breve (4).

Sin embargo, la instalación de la Granja-Escuela en Jerez no fue más que el último de los sucesivos y reiterados intentos que desde la provincia de Cádiz se hicieron para crear un centro de enseñanza y experimentación agraria entre principios y finales del siglo XIX. Entre las iniciativas más interesantes y prometedoras de la primera mitad del siglo hemos de señalar la efimera escuela agraria del Jardín Botánico Príncipe de la Paz de Sanlúcar de Barrameda (1808), y los proyectos frustrados de la Escuela Práctica de Medinasidonia (1841), la Cátedra de Agricultura Práctica de la Junta Provincial de Agricultura (1848) y la Granja-Escuela del diputado jerezano Manuel Bertemati (1855) (5).

Los fracasos de la primera mitad del siglo no desanimaron a los labradores de la campiña que continuaron -ahora estimulados por el mismo Estado- reclamando un centro agrario para la provincia. De las iniciativas y proyectos de la primera mitad del siglo creo haber dado justa cuenta recientemente (6) y ahora me propongo continuar aquella otra historia con otros proyectos e iniciativas de interés que fueron fraguadas en la segunda mitad del siglo: la escuela teórico-práctica de la Diputación de 1872-1873, la granja-modelo del ayuntamiento jerezano de 18811882 y la granja-modelo y estación agronómica del labrador y comisario regio de agricultura Francisco García Pérez. Con ello no pretendo más que subrayar, de una parte, 1) la continuidad del esfuerzo y visión reformadora de un numeroso grupo de labradores de la campiña y, de otra, 2) continuar con mi labor de rescate de personas y proyectos absolutamente desconocidos por la historiografía provincial y, finalmente, 3) aportar nuevas evidencias de que fue sobre todo la ausencia del Estado, siempre empeñado en tareas "más urgentes e importantes" -por lo menos hasta finales de la década del setenta del siglo XIX-, cuando no por su propia ingenuidad liberal -ciega confianza en la iniciativa privada- el que dejó huérfanas a cuantas iniciativas y sugerencias se desplegaron desde Cádiz en pro de un centro de experimentación y de capacitación agraria entre mediados del siglo XIX

(3) E. FERNANDEZ CLEMENTE (1981) y, sobre todo, L. PRIETO (1988 a), (1988 b) y (1992).

(4) Por ahora remito a E. NORIEGA (1911) y Ministerio de Fomento (1912), vol. 1, pp. 214-229.

(5) A. CABRAL CHAMORRO (en prensa).

(6) Ibid. 
y 1887 y por ello, si cabe hablar del "atraso" gaditano, es necesario señalar que sus labradores y campesinos no fueron los únicos responsables.

Tras el fracaso de la Granja-Escuela de M. Bertemati, la primera oportunidad que la provincia tuvo de manifestar su interés por la enseñanza agraria a raíz de la información abierta por el Gobierno por Real Orden de 19 de mayo de 1862.

\section{EL INTERROGATORIO SOBRE EL ESTADO DE LA ENSEÑANZA AGRICOLA, SOBRE LOS MEDIOS DE CONTRIBUIR A SU PROPAGACION Y AL FOMEN- TO DE LA AGRICULTURA, 1862}

Por la Real Orden de 10 de mayo, el Ministerio de Fomento reconocía, de un lado, la labor desarrollada en la agricultura por muchos labradores y ganaderos "inteligentes" y, de otro, el fracaso de medio siglo en la extensión de la enseñanza agrícola y de las granjas-escuelas (7). Para el Gobierno había llegado la hora de concurrir a la labor renovadora con un plan "determinado y fijo", con el apoyo de las localidades y de todas las clases agrícolas del país: creación y organización de escuelas-prácticas de agricultura o de granjas-modelos en "límites razonables" y al menor "costo". Los nuevos establecimientos debían contribuir, mediante "un régimen de cultivo perfecto", no sólo a "manifestar su estado próspero y extender con el ejemplo", sino también a "sobrellevar el peso de los gastos". De todas maneras, pese a este avance de intenciones y de objetivos, la Real Orden dejaba la puerta abierta al señalar que no era la intención del Gobierno sentar doctrinas, que deberían en todo caso nacer de la información abierta, sino manifestar el firme propósito de organizar la enseñanza agrícola sobre "bases sólidas y de provecho positivo".

Desde luego por falta de cuestionario no debió quedar: nada menos que se solicitaron a los pueblos y otras instituciones respuestas a 103 preguntas sobre el siguiente temario $(8)$; 1) si existe escuela o granja-modelo y, en este caso, cuándo fue creada, qué edificios, 'qué terrenos, qué aperos, qué alumnos y cuántos, etc.; 2) si no existen y hubieran de crearse, qué tipo de establecimientos, qué enseñanza, qué cultivos, qué industrias rurales, qué personal, qué máquinas, qué parte de los gastos corresponderían al Estado, etc.; 3) si convendría organizar conferencias agrícolas, misiones agronómicas, bibliotecas agrarias y establecer cátedras de agricultura en las escuelas de veterinaria y qué medidas podrían tomarse en las facultades de ciencias para que éstas colaborasen en la enseñanza agronómica; 5) si convendría crear una sociedad general de agricultura en cuyo caso, qué objeto; 6) si convendría convertir las sociedades económicas en sociedades de agricultura, establecer congresos agronómicos y, finalmente, 8) qué resultados habían producido las disposiciones legislativas sobre la enseñanza agraria en la enseñanza primaria y en los institutos de segunda enseñanza.

(7) La Real Orden puede verse impresa en AMAF (Archivo Municipal de Arcos de la Frontera), leg. 433, "Expediente instruido a virtud de la Real Orden de 10 de mayo de presente año relativa al estado de la enseñanza agrícola y a los medios de propagarla y fomentar la agricultura, 1862" y manuscrita en AMJF (Archivo Municipal de Jerez de la Frontera), leg. 116, "Para contestar un interrogatorio relativo a la enseñanza agraria, 1862".

(8) El cuestionario impreso en ibid y manuscrito en $i b i d$. 
Desde la provincia de Cádiz (9) contestaron al interrogatorio los ayuntamientos de Jerez (10), Medinasidonia, Arcos (11) y Sanlúcar; la Diputación Provincial, la Junta Provincial de Agricultura, la Escuela Industrial y de Comercio de Cádiz y la Normal de esta misma ciudad; las Sociedades Económicas de Jerez y Vejer; la Inspección de Primera Enseñanza, el ingeniero de montes de la provincia y el labrador jerezano Francisco García Pina.

Respecto al tipo de centro, la Diputación, y con ella todos los municipios se inclinaron por el establecimiento de una granja-modelo en Jerez y tres respuestas apuntaron al edificio de la Cartuja. La Sociedad Económica de Vejer y la Escuela Industrial apoyaron que la Granja-modelo de Jerez fuera regional con la inclusión de las provincias de Huelva, Sevilla y Cádiz. El ingeniero de montes, más ambicioso, delimitó una peculiar región agronómica con las provincias de Sevilla, Málaga, Almería, Granada, Murcia, dos terceras partes de Huelva, la mitad de Córdoba, tres cuartas partes de Jaén, dos tercios de Alicante y una pequeña de Albacete.

En cuanto al tipo de enseñanza, la mayor parte de las respuestas propusieron enseñanza de peritos y de capataces y mayorales agrícolas. La Escuela Industrial amplió la enseñanza a la de jardineros y peones agrícolas y el ingeniero de montes incluyó en su respuesta la enseñanza de peritos y de ingenieros agrónomos. Para unos, los alumnos habían de ser pensionados por los partidos judiciales y para otros por los pueblos o las provincias en proporción variable.

Por lo que se refiere a los gastos de instalación, oscilaron entre un millón cien mil reales y medio millón y la gran parte de las respuestas propusieron un reparto de las cargas por mitad entre el Estado y la Provincia. La Escuela Industrial planteó que el Estado corriera con la mitad de los gastos y la otra mitad dividirla en cuatro partes, de las cuales dos corresponderían a Cádiz y las otras dos a partes iguales entre las provincias de Sevilla y Huelva.

Como puede verse, prácticamente había unanimidad en las cuestiones centrales: granja-modelo provincial o regional y gastos compartidos por el Estado y la Provincia. La disonancia la dio el jerezano García Pina. Para éste, las escuelas, como fincas de explotación, esto es Granja-modelo, no eran conveniente porque sus productos "concluirían por desanimar y destruir a los labradores pobres que no podrian sostener las competencias" y, en consecuencia, propuso la erección

(9) Las respuestas de la provincia de Cádiz: AMA (Archivo del Ministerio de Agricultura), leg. 158, "Extracto del expediente sobre el interrogatorio de enseñanza agraria 1862", vol. 1.

(10) Véase también, AMJF, leg. 116 "Para contestar un interrogatorio de la enseñanza agricola, 1862". Asimismo, el períodico local El Guadalete (EG respondió al cuestionario a su manera insertando un artículo que el ingeniero agrónomo y jerezano Eduardo Abela publicó en La Agricultura Española: EG, 12-VII-1862 y una serie completa del mismo sobre un "Plan general de Fomento y enseñanza agrícola" en $E G$ los días $6,13,14,17,20,27$ y 28 de octubre de 1863. Finalmente, señalar que varios años antes, su compañero de estudios y paisano Gumersindo Fernández de la Rosa, publicó en la Revista del Guadalete (Año 1858, pp. 4952) un pequeño trabajo titulado "La agricultura" en el que disertaba sobre la importancia de la enseñanza el progreso agrícola.

(11) Véase también, AMAF, leg. 433 "Expediente instruido a virtud de la Real Orden de 10 de mayo del presente año relativa al estado de la enseñanza agrícola y a los medios de propagar y fomentar la agricultura, 1862". 
de una escuela-experimental que contuviera todas las máquinas, instrumentos y útiles, los diera a conocer mediante ensayo en un pequeño terreno y los prestase a los labradores por una módica retribución.

Por lo demás, la Diputación, la Junta de Agricultura, el Ayuntamiento de Jerez y el director de la Escuela Normal apoyaron las conferencias agrícolas, las misiones agronómicas, bibliotecas y gabinetes de lecturas y la enseñanza agraria en las escuelas primarias e institutos. La Sociedad Económica de Cádiz rechazó las conferencias agrícolas y las misiones agronómicas y la de Jerez elevó el tono y las consideró como "completamente inútiles por sobrar ilustración a los cultivadores de la provincia” y apoyó la celebración de exposiciones agrarias y congresos agronómicos.

La valiosa documentación acarreada por el Gobierno quedó pronto completamente olvidada: Sin embargo, desde la provincia de Cádiz continuaron los proyectos para establecer un centro experimental y de enseñanza. Esta vez la iniciativa corrió a cargo del diputado provincial Antonio María Puelles.

\section{EL PROYECTO DEL DIPUTADO PROVINCIAL ANTONIO MARIA PUELUES, 1872-1873 (12)}

El 10 de diciembre de 1872 el diputado provincial Antonio María Puelles propuso a la Diputación Provincial la erección en la provincia de una Escuela Teórico-práctica de Agricultura. La propuesta, tras el apoyo de los diputados Bozano y Morillo fue aprobada por unanimidad y se encargó a la Comisión de Fomento, de la que el mismo Puelles era presidente, la formulación del oportuno proyecto. La Comisión "sin levantar mano", y en apenas una semana dio por terminado su cometido y el proyecto fue aprobado por unanimidad en sesión de la Diputación de 18 de diciembre (13).

El proyecto constaba de una exposición de motivos, diez capítulos o bases y setenta y tres artículos.

En la exposición de motivos, plena conciencia de los recursos hídricos, agrarios, forestal y pecuarios de la provincia y pesimismo respecto a su estado y desarrollo - del que sólo salva a la viticultura y el vino-, una de las "más atrasadas, cuando debía de ser de las más adelantada, por las buenas condiciones de su clima y de su suelo" (14).

Varias son las razones del atraso: la fecundidad del suelo que hace considerar. como innecesaria la introducción de nuevos sistemas de cultivo e instrumentos, la orientación mercantil y pesquera de la costa, los hábitos de contrabando de los pueblos fronterizos con Gibraltar, la emigración a América, la repulsión a la vidai en el campo, la afición y aplicación de la política y, por encima de todas ellas, la estructura de la propiedad polarizada en torno a los latifundios y minifundios. Pero, a tono con la propuesta, entre las causas fundamentales del atraso de la agricultura gaditana señala la "ignorancia de la clase labriega" (15), tanto de los propietarios como de los trabajadores agrícolas. Unos y otros consideran cual-

(12) A. PUELLES et al. (1873).

(13) Ibid., pp. IV-VI.

(14) Ibid., p. 9.

(15) Ibid., p. 11. 
quier reforma como peligrosa y visionaria y las novedades extranjeras imposibles y dificiles de aclimatar.

El remedio no es otro que acabar con la "ignorancia" y lograr "que la luz de la ciencia económica penetre en los entendimientos, para disipar las tinieblas que lo oscurecen" y de ahí la "urgencia del establecimiento de una Escuela teóricopráctica de Agricultura" (16). Sin embargo, y en contradicción con lo afirmado anteriormente, en la escuela sólo tendrían cabida los hijos de los propietarios, los jóvenes estudiosos y, como acto de caridad cristiana, algunos pensionados huérfanos, pobres y desvalidos.

La base primera del proyecto, titulada "De la escuela" recoge en su artículo uno de los objetivos de la misma: instruir a los jóvenes en el arte de la agricultura, cría y mejora de los animales y el cultivo y la aclimatación y mejora de toda clase de plantas. De manera que la escuela de agricultura de Puelles se articula en un doble plano: de una parte como centro de enseñanza elemental y profesional dirigida a la formación de capataces y peritos agrícolas y, de otro, como centro piloto de orientación y experimentación agrícola.

Como centro de enseñanza, la Comisión de la Diputación no hizo más que recoger, la ley de 11 de junio de 1866 (17) mediante la que se organizó la enseñanza agraria en elemental, profesional y superior. La primera para proveer "capataces" agrícolas con conocimientos meramente prácticos para formar buenos y útiles operarios de agricultura; la segunda para formar "peritos agrícolas" con conocimientos téoricos-prácticos bastantes para tasar, medir tierras y dirigir una explotación agrícola y la tercera destinada a la creación de ingenieros agrónomos que, estudiando la ciencia en su mayor extensión, fueran aptos para dirigir las explotaciones agrícolas, introduciendo los modernos sistemas de cultivos, conforme a las múltiples y variadas exigencias del terreno, del clima y circunstancias económicas de la localidad y como granja su ambición se limitaba a suministrar al público de simientes, plantas y árboles y facilitar a campesinos y ganaderos una parada de sementales de todo tipo de ganados.

En suma, el proyecto de Puelles era más escuela que granja-modelo y más centro de enseñanza que granja de experimentación, y así se recoge en el articulado dedicado en su mayor parte a profesores, cursos y alumnos, y modo respecto a su labor como centro agrario piloto.

La enseñanza de peritos duraría cuatro años y ninguna materia era ajena a su formación: botánica agrícola, agronomía, fisiografía, mecánica, fitotecnia, zootecnia, industria rural, topografia, agrimensura, arquitectura rural y economía rural. La enseñanza de capataces duraría tres años y un programa igualmente completo pero rebajado: botánica, fisiología y patología, mecánica, zootecnia y prácticas de cultivo, injerto, tala y poda.

Para cursar la enseñanza profesional se exigía mediante un examen de ingreso conocimientos de aritmética y álgebra, geometría y trigonometría, física y química, historia natural y geografía y para la enseñanza elemental, leer y escribir correctamente y las cuatro reglas.

(16) Ibid., p. 13.

(17) V.A. MARTINEZ Y MARTIN (1911), p. 5. 
Los alumnos se dividieron en tres clases; pensionados, pensionistas y externos. Los primeros, hospicianos y expósitos, internos y de matrícula gratuita; los segundos, también internos y una pensión-matrícula que no excedería de 750 pesetas por año y curso y los terceros y pago de matrícula, que quedó sin cuantificar.

Respecto a la enseñanza, las clases serían de lunes a sábado, con siete horas a lo menos y nueve a lo más y recomendaba que cada profesor procurara arreglar el programa y las lecciones de manera que coincidieran con las diferentes faenas del cultivo de la granja.

$\mathrm{El}$ personal de la escuela se compondría de un director, al que se le obligaba a residir en la escuela; tres profesores y uno de ellos veterinario; dos ayudantes cuyas funciones serían designadas por el director; dos oficiales, uno de secretaría y otro de administración; cuatro peones encargados; de cuidar el ganado, riego, limpieza y demás faenas; un cocinero, un portero; y un mayoral y un capataz; el primero encargado de los animales y aperos de la granja y el segundo de la explotación de la finca y de las prácticas y ejercicios de campo, así como también responsable del mayoral, de los peones y alumnos. En caso de necesidad, la administración podía recaer en uno de los profesores.

Las titulaciones y condiciones exigidas para aspirar plaza en la escuela iban desde la de ingeniero y peritos para los profesores y ayudantes -a excepción de uno que debía de ser obligatoriamente veterinario-, hasta la de saber leer y escribir correctamente $y$ las cuatro reglas para el capataz y el mayoral.

El presupuesto de funcionamiento de la escuela correría a cargo de la diputación y fue fijado en 125.000 pesetas anuales. No obstante, para el caso de que los ingresos ordinarios de la Diputación no bastasen se invitaría a los pueblos de la provincia y labradores pudientes a que colaborasen y, en el caso de que tampoco este medio diese el resultado apetecido, la Diputación contrataría un empréstito. De todas maneras, el proyecto aspiraba a que la escuela se autofinanciase en su mayor parte con los ingresos de las pensiones de los alumnos, los derechos de matrícula y diplomas y la venta de sus propios productos agrícolas.

La comisión presidida por Puelles propuso que la escuela se instalase en tierras de propios de los pueblos o en tierras pertenecientes a la nación y, a ser posible, en terrenos inmediatos a un monasterio o caserío y para el caso de establecerse en un lugar despoblado ésta podría tomar el carácter de colonia agrícola.

Evidentemente, más que de un proyecto acabado en su totalidad se trataba de unas bases generales o preproyecto a terminar, como propuso la comisión de Fomento, por otra comisión compuesta de diputados provinciales, vocales de la Junta Provincial de Agricultura y de algunas personas competentes en la materia. La misma comisión, encabezada por Puelles, propuso a la Diputación en la sesión en que se aprobaron las bases 1) dirigir una circular a los ayuntamientos que más interés pudieran tener en la erección de la escuela para que reunidos con los agricultores de las localidades respectivas acordasen las cantidades con que pudieran contribuir a la misma; 2) imprimir las bases y enviarla del mismo modo a las localidades para que éstas las tengan presentes y 3) elijan representantes a una asamblea provincial donde definitivamente se apruebe el proyecto (18).

(18) A. PUELLES et al. (1873), pp. V-VI. 
Apenas recibidas las bases, el alcalde jerezano convocó a 47 labradores, 69 viñistas y 2 "capacidades", Manuel Bertemati y Gumersindo Fernández de la Rosa (19); y lo mismo hicieron otros ayuntamientos como los de El Puerto de Santa María (20) o Cádiz (21).

En Jerez, todos apoyaron la idea y se mostraron dispuestos a contribuir "como el que más" al éxito del proyecto. Para la asamblea provincial, que fue convocada para primero de febrero, fueron elegidos el teniente de Alcalde José Oronoz Clemente, el regidor, labrador e ingeniero agrónomo Ramón Guerrero Castro, los labradores, Duque de San Lorenzo, Francisco García Pérez y Miguel Primo de Rivera y Modesto de Castro Solís, viñista (22).

Poco después de la una de la tarde se abrió en los salones de la Diputación la magna asamblea de reformadores agrarios. La asamblea estuvo bien nutrida: sesenta y dos representantes entre alcaldes, regidores, diputados, labradores, mayores contribuyentes y algunos expertos. Presidió el acto el vicepresidente de la Diputación y actuaron como secretarios los diputados Parra y Carrillo (23).

Tras un corto discurso de salutación a cargo del presidente de la Asamblea, Antonio María Puelles glosó las bases de la Escuela de Agricultura y llamó a los concurrentes a que apoyaran el proyecto de erigir la escuela, como única forma de acabar con el "aträso" de la agricultura provincial y "competir con otros mercados".

El proyecto contó con la aprobación de todos. Sólo el diputado Federico Madariaga se empeñó, una y otra vez, en señalar la inviabilidad del proyecto por falta de recursos de la Diputación, cuando no argumentaba defectos de forma. Pronto quedó aislado ante una asamblea entusiasmada y convencida de la bondad de la idea. El debate se centró sobre el lugar más idóneo para el establecimiento de la escuela: en la Cartuja de Jerez o el convento del Valle de esta misma ciudad. Este último se descartó por su lejanía de cualquier núcleo urbano que lo incapacitaba para que pudiera servir de ejemplo a otros labradores, de modo que quedó el convento de la Cartuja. La impaciencia de unos -Santiago Moreno- llevó a proponer la apertura inmediata de la escuela en cualquier local de Jerez y la realización de los trabajos prácticos en fincas cercanas a la ciudad, y la retórica de otros -Jiménez Lozano- llevó a descender de Clavijo a Herrera y de éste a Plinio y Columela. Y, en fin, no faltaron voces más calmadas y reposadas que como la del ingeniero agrónomo jerezano Gumersindo Fernández de la Rosa propusieran "optar por lo posible antes que por lo perfecto" (24).

De todas maneras, algunas de las voces demostraron que no existía una idea acabada y completa de lo que deseaban los concurrentes: si granja-modelo, gran-

(19) AMJF, leg. 116, "Expediente para la creación de una escuela teórico-práctica de agricultura".

(20) AMPSM (Archivo Municipal de El Puerto de Santa María), leg. 186, "Sobre la creación en esta provincia de una Escuela teórico práctica de Agricultura".

(21) EG (El Guadalete), 6-II-1873 y 9-II-1873.

(22) AMJF, leg. 116, "Expediente para la creación de una escuela teórico-práctica de agricultura".

(23) $E G, 6-\mathrm{II}-1873$.

(24) $E G, 6-$ II- 1873 y 9-II-1873. 
ja escuela (25), granja experimental (26) o la suma de todas o de alguna de ellas. Al menos, el ingeniero industrial jerezano García Meneses insistió, con el apoyo de Gumersindo Fernández de la Rosa, en que el centro funcionara como laboratorio y lugar de consulta de labradores y ganaderos, esto es, estación agronómica y granja experimental. Los titubeos de la asamblea en cuanto al tipo de centro a crear no hacian más que reflejar la falta de claridad del mismo gobierno que a la altura de 1873 se movía en un alto grado de indeterminación. Baste recordar que hasta 1882 no se definió claramente los objetivos a cumplir por una estación agronómica y, lo que aún es peor, todavía en 1912 era frecuente la confusión entre las granjas de experimentación y las escuelas prácticas de agricultura (27).

La asamblea aprobó el proyecto y eligió de su seno una comisión formada, en su mayor parte, por jerezanos: García Pérez, Oronoz, Vicente Romero, Castañeda, González de la Mota, García Meneses, Gumersindo Fernández de la Rosa, Lozano Jiménez y: Baltasar Hidalgo (28).

Mientras aquellos entusiastas agrónomos cerraban la asamblea en Cádiz, apenas una semana más tarde, Amadeo de Saboya abdicaba en Madrid y la Asamblea Nacional votaba la proclamación de la república yi fueron ahora los jerezanos quienes tomaron el relevo.

El 25 de mayo de 1874 la Liga de Contribuyentes de Jerez elevó al Ministerio de Fomento un expuesto en el que solicitaba la concesión de la parte del monasterio de la Cartuja que "fueron almacenes y oficinas de labor" para la instalación de la Granja-escuela y adelantaba el compromiso del Ayuntamiento de correr con los gastos de personal y dirección, a cambio de que la Diputación facilitase los terrenos y el material indispensable (29).

La Liga hizo notar al Ministerio la oportunidad del proyecto: mejorar las condiciones de la producción agraria de la provincia y enriquecerla con nuevos frutos y semillas, acrecentar por este medio los ingresos de la Hacienda y conservar el monasterio, una de las "glorias artísticas del país".

En plena vorágine cantonalista y arreciendo la guerra del Norte, la exposición de la Liga de Contribuyentes de Jerez debió quedar para tiempos más tran-

(25) Por granjas modelos o granjas escuelas debe entenderse el tipo de centro que "al paso que se labra la tierra con el objeto de sacar ganancia, se educa e instruye en los buenos principios de la ciencia y en las prácticas del arte a los alumnos o aprendices que con este fin concurren a este tipo de establecimientos". Cf. A. Esteban Collantes A. Alfaro (dir.) (1851), p. 287.

(26) Por el contrario, las granjas experimentales tienen por objeto "plantear sobre el terreno, bajo el concepto industrial, los problemas agrícolas que más interesan a una comarca o región, y ejecutar los experimentos y trabajos de laboratorios para conseguirlo". Cf. "Centro de aplicación de los conocimientos agrícolas", GAMF (Gaceta Agrícola del Ministerio de Fomento), (1886), vol. V, pp. 565-575.

(27) Ministerio de Fomento (1912), p. 70.

(28) $E G, 6-\mathrm{II}-1873$ y $9-\mathrm{II}-1873$.

(29) AMJF, leg. 116 "Expediente para la creación de una Escuela teórico práctica de Agricultura". Véase también el expuesto en $E G, 28-5-1874$ y una pequeña nota en AMA, leg. 135 "Granja modelo en Cádiz, año 1874-1881". La Liga envió asimismo el expuesto al Ayuntamiento jerezano y este fue visto en sesión del Cabildo de 1 de junio de 1874 que acordó enviarlo a la Comisión de policía para que, conjuntamente con el ingeniero Gumersindo Fernández de la Rosa, lo dictaminara. 
quilos. Con ello perdemos todo rastro de nuevos proyectos de granjas-modelos hasta que, encarrilada la Restauración, el Parlamento decretó la Ley de Enseñanza de primero de agosto de 1876.

\section{LA LEY DE PRIMERO DE AGOSTO, 1876: DE LA INFORMACION A LAS CONFERENCIAS AGRICOLAS, 1876-1880.}

Mediante la Ley de primero de agosto, entre otras cuestiones, se ordenó (art. 1.9) la enseñanza obligatoria de una cartilla agraria en todas las escuelas del reino; el establecimiento (art. 2.9) de una cátedra de agricultura elemental en los institutos; la celebración (art. 8) de conferencias agrícolas en todos los pueblos de la monarquía y la posibilidad (art. 6.9) de establecer granjas-modelos, granjasexperimentales y estaciones agronómicas en todas las provincias (30). La Ley de primero de agosto fue acompañada por varias reales órdenes (31) y por la Circular de la Dirección General de Agricultura de 16 de septiembre mediante la que se mandó abrir una -jotra!- amplia información en la que se hiciera constar -para la aplicación de art. 6 de la ley de primero agosto- el parecer de las corporaciones locales y particulares sobre el establecimiento de granjas-modelos y experimentales y muy particularmente sobre los "recursos" que las provincias y pueblos estén dispuesto a dedicar para su sostenimiento (32).

En la provincia de Cádiz, al igual que en el resto de España, la Circular de 16 de septiembre y la Ley de primero de agosto no dejaron más que varios expedientes cumplimentados y un buen número de conferencias magistrales.

Respecto a la información abierta, el Ayuntamiento de El Puerto de Santa María se limitó a contestar lacónicamente sobre la necesidad de establecer una granja en la provincia y defender a su ciudad como el lugar más a propósito (33). La Sociedad Económica de Cádiz, previo informe del socio corresponsal de San Roque, Francisco M. ${ }^{\mathrm{a}}$ Montero, al que por otra parte sigue al pie de la letra (34), se despachó con una decena de folios en los que, de un lado, tomaba nota del atraso de la agricultura de la provincia (arado romano-árabe, pesados y deformes bieldos y toscas y desvencijadas carretas) y, de otro, disertaba sobre la necesidad de la granja-modelo para Cádiz que, en su opinión, era la provincia que más llamada estaba a "patentizar de una manera más clara que ninguna otra los beneficios de estos adelantos (aperos y cultivos) tanto para radicar en ella las mayores y más extensas labores en cotos redondos y las más renombradas y numerosas ganaderías de toda España, como por la templanza de su clima, feracidad de su

(30) Gaceta Oficial, 3 de agosto de 1876.

(31) Véase en la Gaceta Oficial de 17 de agosto las tres Reales Ordenes de 16 de agosto. La primera y la segunda, sobre la reorganización de la enseñanza agrícola en las escuelas primarias e institutos y la organización del certamen para la elección de los libros de textos; la tercera sobre la reorganización de los estudios de la Escuela Superior de Agricultura y la cuarta disponiendo la creación de una Biblioteca Agrícola. Véase también AMA, leg. 158.

(32) La circular en AMJF, leg. 116 "Expediente sobre la creación de Granjas modelo y experimental, 1876".

(33) AMPSM, leg. 186 "El Gobernador Civil de la provincia pide informe a este Excmo. Ayuntamiento, sobre la creación de una Granja Modelo experimental en esta provincia, 1876".

(34) AMC (Archivo Municipal de Cádiz), SEAP, leg. 3.421. 
suelo, diversidad de producciones y ricos y extensos montes". La granja-modelo había de tener como objetivos la formación de capataces y peones agrícolas y patentizar ante los labradores las ventajas de la agricultura moderna. Para la formación de los peones se ideó un sistema singular: la contratación para las tareas de la granja de trabajadores mediante un turno entre todos los de los pueblos de la provincia a propuesta de los ayuntamientos y labradores. Finalmente, los gastos de instalación serían por cuenta de la Diputación (35).

Como ya estamos acostumbrados; el mayor interés por responder a la información abierta por la Dirección General de Agricultura se lo tomó el Ayuntamiento de Jerez (36). Este pasó el asunto a la Comisión de Policía Rural y, asimismo, demandó a la "clase labradora" su propio dictamen.

El informe de la "clase labradora" (37), elaborado por el ingeniero agrónomo Ramón Guerrero Castro y Francisco Orbaneja, reviste, a nuestro juicio, un gran interés y, desde luego, rompe los socorridos tópicos y lugares comunes usados en cuantos informes fueron elaborado entre mediados y finales del siglo XIX y cuyo protagonista fuera el manido atraso de la agricultura española o andaluza. $Y$ es que los labradores jerezanos, abandonados a su suerte por unos gobiernos siempre presto a legislar y poco dados a materializar sus buenas intenciones, hacían más de una década que por propia cuenta y riesgo habían iniciado el difícil camino de la renovación tecnológica de la agricultura de la campiña.

No es por la "falta de conocimientos de los resortes que realizan las mejoras agrícolas, porque en muchos casos no se introducen éstas" sino por la propia complejidad que revisten los cambios en la agricultura que hacen prácticamente imposible que muchos de ellos puedan acometerse por la "iniciativa particular". Los inconvenientes que los labradores señalan quedan muy alejados de la retórica habitual y masoquista de la época: 1) la falta de mano de obra cualificada; 2) la carencia de talleres mecánicos donde se construyan, modifiquen y compongan los intrumentos y máquinas; 3) el desonocimiento sobre qué máquinas y aperos son los mejores y más adecuados para las condiciones agronómicas y sociales de la campiña y 4) la dificultad de relacionarse con los centros industriales donde se fabrican las herramientas y máquinas.

La renovación que reclaman los labradores jerezanos no está exclusivamente sesgada hacia la mecanización, sino que esta la eligen a modo de ejemplo como lo prueba lo que sigue: "lo mismo que decimos respecto a las máquinas, que después de todo no es más que un detalle en las explotaciones rurales, pudiéramos decir de cualquier otro adelanto que se trate de llevar a cabo en la labranza o la ganadería" (38). Por lo tanto el centro que reclaman los labradores es una granja-experimental que dirigida a la realización de ensayos y experiencias pasaran posteriormente a la "común práctica" de agricultores y campesinos. Por lo demás, se muestran dispuestos a contribuir con ganados de labor y rentas y al transporte para el acarreo del material de la granja y construcción de la misma:

(35) Ibid.

(36) AMJF, leg. 116, "Expediente sobre la creación de Granjas modelo y experimental, 1876".

(37) El informe en ibid.

(38) La cursiva es mía. 
La Comisión de Policía Rural del Ayuntamiento jerezano se limitó a suscribir en su totalidad el informe de los labradores y sólo lamentó que por ahora, y vista la situación "aflictiva" de las arcas municipales, como también por lo avanzado del ejercicio económico, le sería imposible al Ayuntamiento coadyuvar a los gastos de instalación y mantenimiento de la deseada granja-experimental (39).

La información abierta por la circular de la Dirección General de Agricultura no dio "resultado alguno" (40) y de la Ley de primero de agosto quedó en la provincia, como ya adelantáramos, la enseñanza agraria -que continuó impartiéndose en las escuelas e institutos- (41) y las conferencias agrícolas.

Respecto a las conferencias agrícolas, ofrecemos un balance, de seguro incompleto, de las pronunciadas en la provincia entre noviembre de 1879 y febrero de 1880 :

CUADRO N.․ 1

Conferencias agricolas en la provincia, 1879-1880

Pueblos N.는 Temas

Alcalá Gaz. 5 Enfermedades del ganado de Cerda; Aparato para la destilación del orujo de la aceituna por el bisulfuro de carbono; La ganadería española; La Philoxera en Gerona y Determinación cuantitativa del aceite contenido en las aceitunas.

Algodonales 11 Enfermedades del ganado de cerda; Las aves de corral; La agricultura en España; Siembra y cultivo de viñas; Cultivos hortícolas; La labor plana y la alomada; Las vides americanas; Nueva enfermedad del olivo; El triunfo de la trilladora a vapor; La constitución de las plantas y Determinación cuantitativa del aceite contenido en las aceitunas.

Barrios, Los 8 Arboricultura frutal; ¿Qué educación agronómica conviene? Aptitudes de los animales; Ideas sobre las exposiciones, concursos de segadoras y exposiciones vinícolas de Navarra y Alicante; La cría de gallos ingleses; Ensayo en Valencia de una nueva legumbre; Las avispas en las viñas y Máquinas para elevar aguas.

Bornos 7 Cultivos hortícolas; El ganado asnal; Razas vacunas; Diferentes abejas que componen una colmena; Un enemigo nuevo en el naranjo; Aljibes o cisternas.

Chiclana $\quad 7$ Enmiendas de suelos; Los caminos vecinales; Poda e injerto; La utilidad para la agricultura de la facilidad en los transportes y Análisis de tierras.

(39) El informe en ibid.

(40) Ministerio de Fomento (1912), p. 65.

(41) M. ${ }^{a}$ D. RODRIGUEZ DOBLA (1989). 
6. Influjo de la agricultura en la civilización; Efectos económicos del grande y pequeños cultivo; Nutrición mineral de los vegetales; Acciones físico-química en los fenómenos de la vegetación; Cultivo de cereales: lo que es y lo que debe ser y El cultivo del Alcornoque.

Conil $\quad 1$ La cuestión de la filoxera.

Gastor, El 1 Siembra y cultivo de cereales.

Jerez :- $\quad 4 \quad$ El cultivo de los cereales; Orden y agricultura e Influencia de la vida animal en los campos.

Olvera : $\quad 1$ El Caballo de silla.

Paterna 2 Discurso inaugural e Idea sobre la filoxera.

Rota 3. Discurso inaugural; La sementera: práctica de la siembra y la zanahoria.

Tarifa 6 Discurso inaugural; Los agentes atmosféricos: su influencia en la vegetación; Tierra vegetal: su composición; El arado: clases y análisis de cada uno de ellos: Los barbechos: ventajas e inconvenientes y Las labores ordinarias en la agricultura.

Trebujena 1 El cultivo de árboles.

Vejer $\quad .10$ Los abonos; Producción y comercio de vinos en España; Conservación de las hortalizas en invierno; Razas vacunas; El barbecho y alternativas de cosechas; La labor plana y alomada; Las ventajas del riego; El arado ordinario en España; El deber de los ganaderos españoles en la actual crisis agropecuaria y El despunte de los brotes de los árboles.

Villamartín 7 . Los fermentos alcohólicos; La: alcachofa; Las vides americanas; Materias colorantes en los vinos y las que se emplean para adulterarlos; Aparatos modernos de molinería; La corrección de mostos y Plantas textiles.

Fuente: Elaboración propia, AMA, leg. 165, "Conferencias agrícolas".

En general, las conferencias, al menos en cuanto a sus contenidos, estuvieron en la mayor parte de las localidades a tono con las posibles necesidades de agricultores y campesinos, aunque desde luego no faltaran casos como el de Los Barrios donde resulta difícil de entender como fue posible que se organizaran unas conferencias sobre las avispas en las viñas, las exposiciones vinícolas de Alicante y Navarra o los gallos ingleses y no incluyeran en el programa la ganadería o la silvicultura del alcornoque y la encina. 
El ejemplo de Los Barrios es indicativo de que aquello se organizó como se pudo, con más buena voluntad que programa y en función de la oferta de conferenciantes y a la verdad es que había poco donde elegir. El peso de las mismas recayó en los profesores de instrucción primaria y mucho nos tememos que no pocos de ellos no hubieran pisado los campos más que en alguna que otra excursión escolar primaveral (42).

En Cádiz brilló la ciencia agronómica. Allí disertaron catedráticos de historia natural, de economía política, de agricultura, de química aplicada; el secretario de la Junta de Agricultura y el ingeniero de montes, eso sí, en una ciudad que no tenía un palmo de tierra que cultivar.

Por el contrario, la ciudad de Jerez contó con un elenco de conferenciantes de talla, algunos de ellos bregados en el campo, y a la medida de su importancia agrícola: el ingeniero agrónomo Gumersindo Fernández de la Rosa disertó sobre, Origen y formación de los terrenos agrícolas, La fermentación alcobólica del zumo de uva, Procedimientos generales de la vinificación: operaciones de vendimia, $y$ preparación de mostos, medios de reforma y mejoramiento, Sobre el cultivo de cereales; el comisario regio de agricultura y agricultor Francisco García Pérez, sobre Orden y agricultura; el ingeniero de montes Adolfo Parada y Barreto, sobre Influencia de la vida animal en el campo; los catedráticos del Instituto Provincial Santos Landas, Juan María San Juan y Juan Argullós, sobre Meteorología agrícola, Bellezas y ventajas de la vida del labrador en el campo, Fisiología vegetal y Empleo del orujo de la uva en la producción de vinos baratos; el médico Domingo Grondona, sobre la Influencia de la agricultura en la salud de los pueblos y el doctor en derecho administrativo Eduardo Badia, sobre la Industria vinicola; sus actuales condiciones económicas y medios de mejorarla (43).

El punto final a las conferencias agrícolas en la provincia debieron de ponerlo los mismos conferenciantes, cuando no el público para las que fueron creadas. En no pocos casos -entre ellos Algodonales, Vejer, Bornos, Los Barrios y Villamartín- la nómina de conferenciantes sólo incluía al único maestro de instrucción, que acabaría por desertar silenciosamente; en otras -Alcalá del Valle, Jimena, Medina y Puerto Real-, nunca llegaron a celebrarse por la "falta de público" y no faltó localidad donde surgieran conatos de rebelión -como el caso de Jerez donde el alcalde amenazó al claustro de profesores del Instituto Provincial (44)contra los artículos 8 y 9 de la Ley de $1 .^{\circ}$ de agosto de 1876 por lo que, de un lado, se obligaba a los catedráticos, ingenieros y funcionarios públicos que "cobran sueldo del Estado" a explicar una conferencia y, de otro, ordenaba a los

(42) En Algodonales, Los Barrios, Bornos, Conil, El Gastor, Olvera, Rota, Tarifa, Trebujena, Paterna, Vejer y Villamartín fueron pronunciadas por los maestros de escuelas de la localidad. Sólo en los casos de Alcalá de los Gazules, Chiclana, Jerez y Cádiz intervino algún que otro propietario agrícola o abogado o algún que otro ingeniero a catedrático.

(43) Véase, AMJF, leg. 116, "Sobre la celebración de las conferencias agrícolas, 1876" y Bandos Municipales, años 1876-1880 y AMA, Leg. 165, "Conferencias agrícolas" y leg. 168 "Enseñanza agrícola". En el expediente jerezano citado más arriba puede verse el programa de conferencias agrícolas diseñado por la Junta de Agricultura y de Instrucción Pública en reunión celebrada conjuntamente con el ayuntamiento de la ciudad.

(44) AMJF, leg. 116, "Sobre la celebración de las conferencias agrícolas, 1876". 
maestros, a falta de otra persona, a leer en todo los pueblos un "capítulo de la obra" que designara la Junta de Agricultura, Industria y Comercio. A comienzos de 1880 las conferencias agrícolas pasaron a mejor vida.

Sobre el eco de las conferencias agrícolas poco he podido averiguar: el Ayuntamiento de Jerez alquiló cien sillas para el acto inaugural, pero tampoco sé si aquellas llegaron a ocuparse (45). Quizás el resultado más positivo de las conferencias fuera reunir durante algunos meses, y dos años en el caso de Jerez, a buen número de labradores y algún que otro pequeño y mediano campesino en torno a una misma mesa en la que discutir sobre las dificultades más apremiantes de la agricultura local y provincial. Sirva también la celebración de las conferencias gaditanas para probar cómo los pueblos se aprestaron a colaborar con generosidad con la pobre iniciativa del Estado.

Por conferencias no debió quedar, y si desde luego la renovación de la agricultura de la campiña gaditana hubiera dependido de su celebración esta era cosa hecha. El mismo año de 1879 en que las conferencias agonizaban, la Dirección General de Agricultura se aprestaba (R.O. 19 de 1879) a acabar con la filoxera mediante la celebración de tres disertaciones sobre el insecto en todas las provincias de España (46). Para el caso de Cádiz, dos de las dichas conferencias filoxéricas se celebraron en Jerez y fueron sus ponentes el ingeniero de montes Adolfo Parada y Barreto (47) y el ingeniero agrónomo Gumersindo Fernández de la Rosa (48).

Peor resultado que el de las conferencias agrícolas aguardó al artículo 6.9 de la Ley de primero de agosto por el que se preveía la creación de granjas escuelasmodelos, experimentales en todas las provincias. Que sepamos, el artículo durmió en el sueño de los justos y con él cualquier otra iniciativa gaditana respecto a las granjas escuelas hasta la Real Orden de 9 de mayo y los Reales Decretos 14 de mayo y 23 de septiembre de 1881 por los que se crearon las granjas-modelos de Granada, Sevilla, Valladolid y Zaragoza.

\section{DE LA REAL ORDEN DE 9 DE MAYO DE 1881 AL PROYECTO DE GARCIA PEREZ DE 1885: EL FIN DE LA OBSESION PEDAGOGICA}

La Real Orden de 9 de mayo de 1881 se limitaba a anunciar la intención del Gobierno de promover una campaña activa en favor de la agricultura, la industria y el comercio mediante la enseñanza agraria, la realización de un mapa agronómico, la repoblación forestal y el establecimiento de granjas-modelos y de esta-

(45) AMJF, LAC, año de 1877, Acta de 2 de enero, 12 de mayo, 27 de junio, 16 de julio y 16 de agosto de 1877 .

(46) Sobre la filoxera en la provincia de Cádiz preparo un trabajo que daré a conocer en su momento.

(47) A. PARADA Y BARRETO (1880).

(48) F. FERNANDEZ DE LA ROSA, "Historia de la plaga filoxérica, y sus estragos en los países vitícolas de Europa. Crítica de los medios de curación intentados. Medidas preventivas que en nuestra localidad deben adoptarse", Revista de Montes, 90 de los medios de curación intentados. Medidas preventivas que en nuestra localidad deben adoptarse", Revista de Montes, 90 y 91 (1880), pp. 457-466 y 481-494. Un resumen de la conferencia en $E G$, 29 y 31 de julio de 1879. Véase también, AMAPA, leg. 168. 
ciones agronómicas (49). Y por el Real Decreto de 14 de mayo (art. 4.9) se comprometió el Gobierno a auxiliar "con el material necesario y con el personal facultativo a tres provincias que antes del 15 de junio próximo soliciten el establecimiento de estaciones agronómicas o granjas modelos, debiendo consignarse en las solicitudes las cantidades que destinan para los gastos anuales de dicha institución y las condiciones de la finca en que ha de instalarse" (50).

La Diputación Provincial decidió acogerse al citado decreto y elevó en fecha de 10 de junio una exposición en ese sentido (51). La Diputación argumentó la condición agrícola de la provincia y el abatimiento de su agricultura. Respecto a su compromiso económico se remitió a una posterior reunión que, por supuesto, no tuvo lugar ni en ese año ni en los siguientes (52).

El Real Decreto de 14 de mayo empalmó, sin solución de continuidad, con otro de 23 de septiembre del mismo año. Por este Decreto el Gobierno decidió dar un paso hacia adelante y tomó la iniciativa al crear tres granjas modelos en cada una de las provincias de Sevilla, Granada, Zaragoza y Valladolid (53). Varios días antes de que se firmara el decreto se vio en el Cabildo de Jerez una carta del diputado José Sánchez Mira en que éste animaba al Ayuntamiento a solicitar del Ministerio de Fomento una de las granjas previstas. El Cabildo apoyó la idea, designó una comisión, adelantó como posible lugar de su ubicación el Hato de la Carne (terreno comunal) y acordó elevar un expuesto en ese sentido (54).

En 23 de septiembre elevaron la exposición (55). En ella recuerdan que era éste un proyecto largamente acariciado, la anterior exposición de la Diputación, la importancia de Jerez como centro agrícola y, desde luego, la crisis agropecuaria y vinícola que amenazaba con llevarse por delante el "renombre y opulencia" del centro agrícola y comercial jerezano. Fomento exigió al Ayuntamiento que concretase la cantidad con la que se estaba dispuesto a contribuir a la erección de la granja modelo y, asimismo, sugirió que la granja fuera local (56).

(49) Gaceta de Madrid, 10 de mayo de 1881.

(50) Ibid., 18 de mayo. Otras medidas previstas en la Real Orden fueron las que siguen: publicación en la Gaceta de Madrid de resúmenes mensuales de cuantos trabajos se hubieran practicado en la Escuela General de Agricultura; redacción de una memoria anual por dicha escuela y el compromiso del Gobierno de dar a la citada memoria la mayor publicidad; celebración semestral en la Escuela de un concurso de máquinas agricolas; propósito del Gobierno de llevar a las Cortes para su aprobación de una partida destinada a premiar a las dos fincas "mejor cultivadas" y al agricultor que hubiera construido edificios a mayor distancia de poblado y, finalmente, creación de un premio anual a la mejor obra escrita de agricultura.

(51) AMA, leg. 135, "Granjas modelos en Cádiz, años 1874-1875". La rapidez con que la Diputación elevó el expuesto hizo innecesario un oficio de la Junta Provincial de Agricultura de 3 de junio en el que instaba a la Diputación a reclamar una de las granjas. Véase: $A D P C$ (Archivo de la Diputación Provincial de Cádiz), LAD (Libros de Actas de la Diputación), año 1881, Acta de 29 de junio.

(52) Me baso para ello en ADPC, LAD, años 1881-1883. De nada sirvió tampoco la petición de la Junta Provincial de Agricultura reclamando una consignación para la futura granja en los presupuestos. Véase: ADPC, $L A D$, año 1881, Acta de 29 de junio.

(53) Gaceta de Madrid, 24 de septiembre de 1881.

(54) AMJF, LAC año de 1881, Acta de 21 de septiembre.

(55) AMA, leg. 135, "Granjas modelos en Cádiz, años 1874-1881".

(56) AMJF, LAC año 1881, Acta de 31 de octubre. 
El proyecto de una granja modelo para Jerez se cruzó en el camino con la necesidad de los ganaderos de contar con un nuevo recinto para feria de ganados, de modo que se unificaron ambos proyectos en el titulado "Ante-Proyecto... para la instalación de la Feria de ganado y Granja Modelo en la Era de Modelos" (57) y para cuya realización se aprobó por Real Orden de 31 de julio de 1882 un empréstito municipal (58).

La unificación de la feria de ganados y la granja modelo fue el final de la historia. Dificultades y diferencias de última hora entre el Ayuntamiento, ganaderos y labradores en cuanto al lugar definitivo para la ubicación de la feria terminaron por prolongar el asunto. Entre tanto, algunos labradores jerezanos poco confiados -y con razón- y viendo que una vez más la granja se les iba de las manos se aprestaron por su cuenta y por última vez a proyectar para Jerez una granja modelo privada, con el apoyo de la Diputación Provincial. Esta vez fue obra del labrador jerezano y comisario regio de agricultura Francisco García Pérez.

A finales de mayo de 1884 se inauguró la colonia agrícola de Campano (Chiclana) del marqués de Bertemati, con la asistencia de un numeroso público (59). En un clima de euforia agrarista y modernizadora, la inauguración fue aprovechada por el comisario regio para exponer su proyecto de una estación agronómica para la provincia. El proyecto fue recogido, a sugerencia de García Pérez, por varios diputados provinciales. La Diputación se mostró de acuerdo y eligió la oportuna comisión -iotra!- para estudiar el asunto que quedó ultimado en abril del año siguiente (60).

El proyecto de García Pérez era dobie: granja modelo y estación agronómica. La primera dirigida a resolver los problemas del desarrollo práctico de la agricultura y de la ganadería progresiva y la enseñanza de obreros. La segunda, centro de investigación, de ensayos y de consulta técnica para los labradores.

Acorde con estas funciones, la granja-modelo había de contar con una finca de proporciones adecuadas y adelanta la propiedad de Juan Pedro Aladro situada entre Jerez y El Puerto de Santa María, a orillas del Guadalete con 100 a 150 hectáreas de pan sembrar; 25 o más de viña y arboleda, abundantes aguas y buenos edificios. Por el contrario, para estación agronómica bastaba una finca de 10 hectáreas situada en las cercanías del pueblo con terrenos adecuados para los ensayos de todo tipo y un caserío capaz de servir para laboratorio químico-agrícola, gabinete de observación metereológica y almacenes para los productos, maquinaria y dependencias menores.

Consciente García Pérez de las dificultades presupuestarias de la Diputación, propuso como recursos para llevar a cabo el proyecto el establecimiento

(57) G. FERNANDEZ DE LA ROSA, "Ante-Proyecto formulado por el ingeniero agrónomo titular para la instalación de la Feria de ganado y Granja modelo en la era de Morales", EG, 4-II1882. Véase también AMJF, LAC año 1881, Acta de 7 de noviembre.

(58) AMJF, leg. 302, "Sobre traslación de la Feria de Ganados a las inmediaciones de la población en terrenos de la Era de Morales, 1899".

(59) $E G, 28$-V-1884.

(60) F. GARCIA PEREZ (1885) y ADPC, LAD, año 1884, Acta de 7 de junio. Un resumen en $G A M F$, (1885), tomo 1, pp. 719-720. La formación de la comisión en ADPC, LAD año 1884, Actas de 19 de junio y 30 de noviembre de 1884 . 
de una sociedad anónima con un capital de 400.000 reales divididos en acciones de 2.000 reales a cuyo capital social había que sumar los auxilios que el Gobierno concedía a este tipo de establecimientos, una subvención anual de la Diputación de 25.000 pesetas y otra cantidad que no fija del Ayuntamiento jerezano.

En la proyectada sociedad anónima hay algo más que falta de recursos públicos. Hay también, y mucha, desconfianza en el papel del Estado: la granja y la estación habían de interesar a los agricultores y sobre todo a los propietarios para hacer de ella "un negocio de explotación" y evitar de esta forma que éstas se convirtieran en un plantel de "empleados políticos" y es que, como el mismo proyecto señala, el "interés individual es más poderoso, más eficaz y ejecutivo que el oficial".

El proyecto, más bien anteproyecto, no entró en realizar un presupuesto detallado de los gastos de instalación y funcionamiento de la granja, aunque sí el de la estación:

\section{CUADRO N. 2}

\section{Presupuesto de instalación y gasto anual de la estación agronómica}

\begin{tabular}{|c|c|c|c|}
\hline Gastos de instalación & Pesetas & Gasto anual & Pesetas \\
\hline -Máquinas y enseres de cultivo & 18.500 & -Arrendamiento del local y terrenos & 4.000 \\
\hline -Material de laboratorio & 1.000 & $\begin{array}{l}\text {-Entretenimiento del gabinete } \\
\text { de ensayos químicos }\end{array}$ & 1.500 \\
\hline -Material del observatorio & 1.500 & $\begin{array}{l}\text {-Entretenimiento del observatorio } \\
\text { metereológico }\end{array}$ & 500 \\
\hline \multirow{6}{*}{$\begin{array}{l}\text {-Menaje de la casa habitación } \\
\text {-Reformas de adaptación } \\
\text { del edificio } \\
\text {-Preparación de los terrenos } \\
\text { y obras permanentes de } \\
\text { mejora y seguridad }\end{array}$} & 2.000 & -Conservación del mobiliario mecánico & 1.000 \\
\hline & 1.500 & $\begin{array}{l}\text {-Capital circulante (semillas, abonos, } \\
\text { labores, etc.) }\end{array}$ & 5.000 \\
\hline & & $\begin{array}{l}\text {-Personal administrativo para el } \\
\text { cultivo y contabilidad }\end{array}$ & 3.000 \\
\hline & 1.000 & $\begin{array}{l}\text {-Personal facultativo (director y } \\
\text { ayudantes) }\end{array}$ & 8.000 \\
\hline & & -Imprevistos & 1.000 \\
\hline & 25.000 & & 24.000 \\
\hline
\end{tabular}

El proyecto de García Pérez no pasó del papel. A comienzos de mayo, tras el dictamen de la Comisión de Fomento (!!), el Pleno de la Diputación se limitó a recoger lacónicamente en sus actas el aplazamiento de su ejecución "por los gastos que ha de originar hasta tanto que el tesoro provincial se encuentre más desahogado" (61). Con ello hemos llegado al final de nuestra historia a la que sólo restan las conclusiones.

(61) ADPC, LAD año 1885, Acta de 8 de mayo. 


\section{CONCLUSIONES}

Entre 1860 y 1887 fueron sucesivos y reiterados los intentos del Estado por coadyuvar a la renovación de la agricultura española mediante la extensión de la enseñanza agraria y la erección de granjas-modelos y granjas-escuelas a todas las provincias. Desde Cádiz -al igual que desde otras provincias andaluzas- (62) no faltaron ni intentos autoctónomos de enseñanza agraria (proyecto de Puelles y de la Diputación) ni estaciones agronómicas (proyecto de García Pérez). Asimismo, mostraron los gaditanos su interés por secundar las iniciativas del Gobierno tanto en las informaciones abiertas (1862 y 1876) como en las conferencias agrícolas (1876). Sin embargo, nuestra historia es la historia de un fracaso.

Dos son las razones que a nuestro juicio hicieron imposible la creación de una granja-escuela: En primer lugar, la timidez y tacañería de los sucesivos gobiernos que a la par que mandaban erigir -con desmedida ambición- unos centros en los que ni tan siquiera faltaba como personal un capellán sólo se comprometía a costear el personal facultativo, reducido a un ingeniero y un perito (63); en segundo lugar, la falta de adecuación de las propuestas del Gobiemo con las necesidades de los labradores de la provincia: mientras el primero se empeñaba en las granjas-escuelas, los segundos reclamaban más (sobre todo los jerezanos) un centro de experimentación o si se quiere una granja-experimental, de manera que los proyectos de granjas escuelas espoleados por el Gobierno encontraron un muy escaso eco real en la provincia.

Y llegado aquí convendría plantear el porqué de la obsesión del Gobierno por las granjas-escuelas y el olvido por los centros de experimentación agraria. Sobre este particular varias son asimismo las razones. En primer lugar, por la larga tradición dieciochesca, demasiada apegada a la teoría y a la educación (64); en segundo lugar, por el mimetismo de los modelos franceses (65); en tercer lugar, porque siempre podría cubrirse el expediente ordenando la enseñanza de la agricultura en la escuela elemental y secundaria sin gravar los presupuestos del Estado y, en cuarto lugar, por cierto doctrinarismo liberal que llevó a confiar excesivamente en la iniciativa privada.

Pero las responsabilidades no sólo fueron del Gobierno: los ayuntamientos de los pueblos $y$, ante todo, la Diputación siempre acompañaron con gusto la

(62) Para Sevilla: M. Parias Sáinz de Rozas (1983 a) y (1983 b). Para Córdoba: F. Amor Mayor (1858). Para Málaga: L. Prieto Borrego y J.L. Casado Bellagarza (1994). También AMA, Leg. 127.

(63) El Real Decreto de 23 de septiembre de 1881, por su artículo 5. señalaba que cada granjaescuela había de tener un director; un ayudante, un veterinario, un capellán, un médico, un conserje, personal subalterno de capataces, mayores, aperadores, hortelanos, jardineros y los peones que fueran necesarios y sólo "los sueldos de los dos primeros funcionarios se consignarán en los presupuestos generales del Estado. Los restantes serán a cargo de las provincias respectivas".

(64) A. CABRAL CHAMORRO (en prensa).

(65) G. CESALDO, "La enseñanza de la agricultura en Francia y el Instituto Agronómico", GAMF, V (1876), pp. 160-172, 274-283 y 438-448. Véase también, E. Fernández Clemente (1990). 
tacañería gubernamental (66). Sólo cuando el Gobierno, espoleado por la crisis agropecuaria, mediante el Decreto 9 de diciembre de 1887, se comprometió, de un lado, a correr con todos los gastos de "sostenimiento" de las granjas escuelas experimentales desde el momento de su instalación y, de otro, se dio un giro de ciento ochenta grados en los objetivos de los centros -más experimentación que escuela y en todo caso "escuela" de capataces y de obreros- se dieron las condiciones óptimas para hacer realidad en la provincia los anhelos de cincuenta años y hacer florecer como hongos el entramado institucional (granjas de experimentación, estaciones vitícolas, institucional (granjas de experimentación, estaciones vitícolas, enológicas, antifiloxéricas, etc.) que está detrás de la recuperación, nuevo rumbo y desarrollo de la agricultura española entre finales del siglo XIX y primer tercio del siglo XX.

Sin embargo, el tiempo no transcurrió en balde y del mismo modo que las granjas experimentales, estaciones agronómicas, enológicas, vitícolas, etc. supieron vencer algunos de los obstáculos de la agricultura española de finales del siglo XIX, la ausencia de estas durante las décadas centrales de ese mismo siglo son factores que ayudan a explicar las dificultades de renovación con que se encontraron los labradores y campesinos españoles entre mediados del diecinueve y mil novecientos.

(66) No sólo la Diputación se mostró ruin con los proyectos de escuelas, granjas y estaciones sino que tenía herida de muerte desde sus orígenes a la mismísima Junta Provincial de Agricultura. El ingeniero agrónomo de la provincia Domingo Lizaur resume su historia entre 1875-1885: "por reglamento corresponde a la Diputación Provincial dotar al Consejo Provincial de ambos elementos (material y personal) y desde un principio, fuerza es decirlo, se mostró mezquina de sus atribuciones. Primeramente el personal que asignó se componía de un auxiliar, dotado de 1.500 pesetas anuales, un escribiente y un mozo-portero con 750 , y el material 375 pesetas; pero bien pronto se cansó de tanta largueza, y por razón económica en su presupuesto, se suprimió primero el auxiliar, reduciendo el material a 125 pesetas solamente. Con posterioridad vuelve a crear la plaza de auxiliar y suprime la de escribiente, aumentado por último el capítulo de material a 250 pesetas al año. Dotación que como se ve no puede ser más mezquina ni en personal, ni en material... Mas no sólo ha sido la escasez de personal y de material lo que ha impedido el buen servicio de la Corporación: el continuo cambio de personal, en su mayoría poco idóneo, la falta de exactitud en el pago hasta de ese exiguo material, ha sido moneda corriente, dificultando más y más la marcha de este Consejo Provincial, que en ocasiones ha carecido hasta de papel en que formular sus escritos". Véase: AMA, leg. 257, "Memoria redactada por el Ingeniero Agrónomo Secretario del Consejo Provincial de Agricultura, Industria y Comercio de Cádiz en cumplimiento de orden circular del Consejo Superior del ramo, fecha 14 de julio último". 